\title{
ANISOTROPIC ULTRASONIC BACKSCATTER FROM THE RENAL CORTEX
}

\author{
Jonathan M. Rubin, Paul L. Carson and Charles R. Meyer \\ Department of Radiology, University of Michigan Medical Center, Ann Arbor, MI 48109, U.S.A.
}

(Received 28 October 1987; in final form 4 February 1988)

\begin{abstract}
We have demonstrated a significant, directionally dependent, anisotropic, echogenicity from the cortices of two sheep kidneys and a normal human cadaver kidney. The anisotropy corresponds to the relationship of the sound field to the medullary rays and interlobular arteries. At $7.5 \mathrm{MHz}$, the backscatter from specimens of cortex of a sheep kidney was $4.7 \pm .7 \mathrm{~dB}$ (mean \pm SEM) greater in parts of the cortex where the field was perpendicular to these structures as opposed to where it was parallel to them. In addition, we measured the angular distribution of this anisotropy and compared it to the $5 \mathrm{~dB}$ inclusion angle of the $5 \mathrm{MHz}$ linear array that was used in measurements on the whole kidney specimens. The transducer inclusion angle was $10^{\circ} \pm 2^{\circ}$, while the angular distribution was $34^{\circ} \pm 22^{\circ}$ and $23^{\circ} \pm 11^{\circ}$ (mean \pm SD) for the intact sheep and human kidney, respectively, demonstrating some variation of the medullary rays from perfect specular reflectors. This anisotropy should be visible in standard diagnostic scanning, and its recognition could increase ultrasound's sensitivity for the detection of renal disease.
\end{abstract}

Key Words: Ultrasonic backscatter, Renal cortex, Kidney, Tissue characterization.

\section{INTRODUCTION}

When diagnostic ultrasound scans are performed, a standard assumption is made that reflections from an organ are independent of the directions from which they are scanned. For many organs, i.e. the liver, this approximation is probably a good one (Fei and Shung, 1985), but there are tissues from which the echogenicity varies depending on their orientation relative to the sound beam. This property, known as anisotropy, has been observed in the heart, tendons, and skeletal musculature (Fei and Shung, 1985; Miller et al., 1983; Mol and Breddels, 1982; Ophir $e t$ al., 1982; Cady et al., 1983; Fornage, 1986; Fornage, 1987), and although diffraction patterns have been studied in many tissues (Nicholas, 1977, 1979; Chivers, 1981), a visually discernable anisotropic backscatter has been alluded to, but never demonstrated, in abdominal organs (Fei and Shung, 1985).

We have demonstrated a significant anisotropic effect in reflections from the renal cortex of two sheep kidneys and a normal human cadaver kidney. This effect was quantified in one of the sheep kidneys

Address further communication to: Jonathan Rubin, M.D., Department of Radiology, B1 D502, University of Michigan Medical Center, Ann Arbor, MI 48109-0326. using a specially designed acquisition system and tissue characterization programs.

As gray scale imaging has continued to develop and with the improved resolution of modern ultrasound scanners, ultrasound's ability to detect renal abnormalities has also increased. We suspect that the recognition of this anisotropy will further aid in the detection of renal pathology.

\section{MATERIALS AND METHODS}

Three specimens were scanned in vitro, including two kidneys from freshly slaughtered sheep and a kidney from a 19-year-old female with a ventricular septal defect, pulmonic stenosis, and right ventricular hypertrophy. The autopsy showed a congested but otherwise normal kidney.

The specimens were scanned in normal saline on sound absorbing material, cloth toweling or a pad of butyl rubber. Imaging was performed using either 5 $\mathrm{MHz}$ linear or sectored array scanheads (Acuson 128), or for the quantitative measurements, scanned with a $7.5 \mathrm{MHz}$ fixed focus transducer (ATL Mark 100). One of the sheep kidneys and the cadaver kidney were scanned intact. The organs were scanned sequentially in transverse planes using both multiple 
and single focal zones with the phased array transducers. The kidneys were then rotated $90^{\circ}$ and rescanned in a similar manner. This rotation changed the orientation of the renal architecture to the sound field. The time-gain compensation (TGC) curve was flattened to remove or at least standardize any variation in sensitivity with depth, and the curve was identical before and after the rotation.

To further control for the effects of machine settings, a homogeneous, isotropic cardiac phantom of approximately the same cross-sectional dimensions as a kidney was scanned in saline. The phantom was a truncated cone with thick walls, approximating the shape of a heart chamber in axial views. This phantom had small scattering spheres embedded in it, and it was composed of urethane rubber with a speed of sound equal to and an attenuation coefficient and backscatter coefficient approximately equal to that of soft tissue. Images of the phantom were compared to those of the intact renal specimens to evaluate the effects of overall gain and of focal zone, attenuation and other possible changes in relative sensitivity (Fig. 3).

The relative backscatter measurements were performed on fresh sheep kidney at room temperature in normal saline using a $7.5 \mathrm{MHz}$ scanhead on the ATL Mark 100 ultrasound scanner. The transducer had a geometric focus at $2.1 \mathrm{~cm}$ with a range of $1-4 \mathrm{~cm}$. Renal cortex was dissected out under normal saline and glued to a butyl rubber support in a saline scanning tank using cyanoacrylate. The butyl rubber was in a cylindrical shape with a radius of curvature of $4.5 \mathrm{~cm}$. Tissue samples, typically 0.7 to $1 \mathrm{~cm}$-thick were scanned at a range of $2-3 \mathrm{~cm}$ from the transducer. A sample with the radial structures perpendicular to the beam was placed beside a sample with these structures parallel to the beam (Fig. 4).

The RF signal was digitized in real time at 25 $\mathrm{MHz}$ using a Precision Data Systems digitizer with digital storage and control on a dedicated Nova $4 \mathrm{X}$ minicomputer (Meyer et al., 1985). Regions for backscattering analysis were selected in the tissues with structures parallel and perpendicular to the beam. In both tissues, the analysis regions began at the same distance from the transducer and were of equal axial length. Tissue regions were chosen such that the amount of overlying tissue prior to the start of the region was the same within 1 or $2 \mathrm{~mm}$.

The envelope of each RF line was detected by the Hilbert transform and a mean envelope was obtaine. for each tissue orientation in the image. The mean envelope from tissue with one orientation was subtracted from that with the other. This resulting logrelative backscatter as a function of depth was fitted to a straight line to correct for differences in attenuation coefficient in the two tissue orientations. Relative backscatter was taken as the value in $\mathrm{db}$ of this log-relative difference line at the start of the analyzed sample volume. This method of taking the relative backscatter differs from that described by O'Donnell et al. (1979) in cardiac tissues, in that O'Donnell, takes the mean relative backscatter throughout the entire sample volume. With relatively thin tissue samples, differences in the two methods should be small (O'Donnell et al., 1979).

Finally, to characterize more accurately the reflectors producing the anisotropy, the inclusion angle of the region of increased echogenicity was measured in both intact specimens. A standard goniometer was used to measure the angle of the visually discernable hyperechoic region directly from images of both kidneys made with the $5 \mathrm{MHz}$ linear phased array scanhead. Multiple measurements were made, 15 from the sheep's kidney and 29 from the cadaver kidney. These were compared with the $5 \mathrm{~dB}$ inclusion angle for a specular reflector using the $5 \mathrm{MHz}$ linear array probe with multiple focal zones.

\section{RESULTS}

Figures 1 and 2 demonstrate typical slices through the intact sheep and human kidneys. There are clearly demarcated areas of increased echogenicity in both kidneys where the cortex is perpendicular to the sound field. After rotating each kidney $90^{\circ}$, the areas of increased echogenicity changes, but the orientation of the echogenic areas to the sound field does not change (Figs. 1 and 2).

A typical cross section through the cardiac phantom showed generally uniform echogenicity throughout except in the far field where there was a fall off due to attenuation (Fig. 3). No anisotropy was demonstrated. Further, no local areas of increased echogenicity along lines corresponding to the ranges of electronic focussing were seen (Fig. 3). Hence, the observed increased echogenicity was not due to focal zone effects.

The relative backscatter was determined for scattering perpendicular and parallel to the radially oriented structures in dissected fragments of a sheep's kidney. Measurements on 18 different segments revealed backscatter an average $4.7 \mathrm{~dB}$ higher when the tissue structure is normal rather than parallel to the ultrasound beam (Fig. 4). The standard error of the mean of these 18 measurements was $0.7 \mathrm{~dB}$. This is probably a lower limit on the difference, since it was not always possible to be oriented exactly perpendicular to the radial tissue structures. 


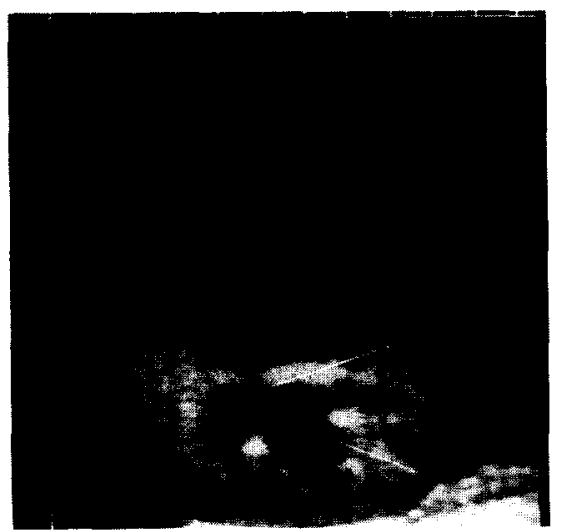

(a)

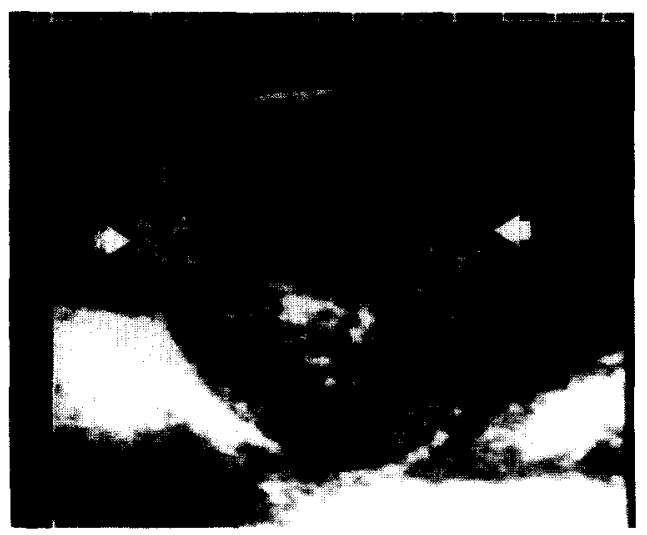

(b)

Fig. 1. (a) Fresh sheep kidney scanned with multiple focal zones using the $5 \mathrm{MHz}$ linear array scanhead. There are areas of increased echogenicity noted in the cortex at 3 o'clock and 9 o'clock corresponding to where the medullary rays and interlobular arteries would be perpendicular to the sound field. The two intersecting white lines define the inclusion angle that approximately inscribes the area of increased echogenicity on the right-hand side of the cortex. A similar angle was measured for the left-hand side as well, producing two inclusion angles per image. (b) The same sheep kidney imaged in 1(a) after having been rotated $90^{\circ}$. Two new areas in the cortex are now perpendicular to the sound field and are now hyperechoic (arrows).

The inclusion angles of increased cortical echogenicity in the intact sheep and human cadaver kidney were $34^{\circ} \pm 22^{\circ}$ and $23^{\circ} \pm 11^{\circ}$ (mean \pm standard deviation of the sample), respectively. The $5 \mathrm{~dB}$ inclusion angle for echoes from a flat surface with the 5 $\mathrm{MHz}$ linear array transducer was $10^{\circ} \pm 2^{\circ}$ (mean \pm standard deviation).

\section{DISCUSSION}

The clinical interpretation of echogenicity is the benchmark for the diagnosis of renal disease. The normal renal cortical echogenicity in adults is generally considered to be less than that of the normal liver and the renal sinus (Rosenfield et al., 1980; Rosenfield et al., 1978; Cook et al., 1977; Marchal et al., 1986). Only in young infants is the echogenicity of normal cortex equal to or greater than that of the liver (Haller et al., 1982; Hricak et al., 1983; Cramer et al., 1986). Otherwise, variations in the echogenicity of the renal cortex suggest the presence of disease; particularly, a generalized increased echogenicity is a

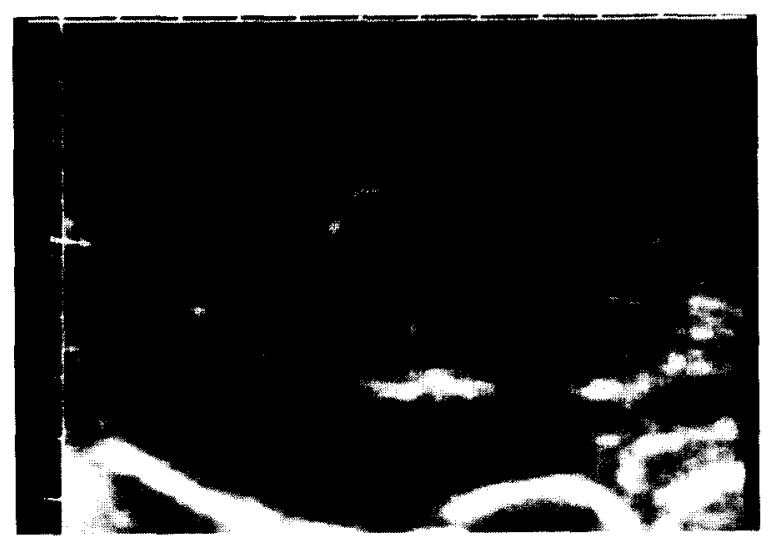

(a)

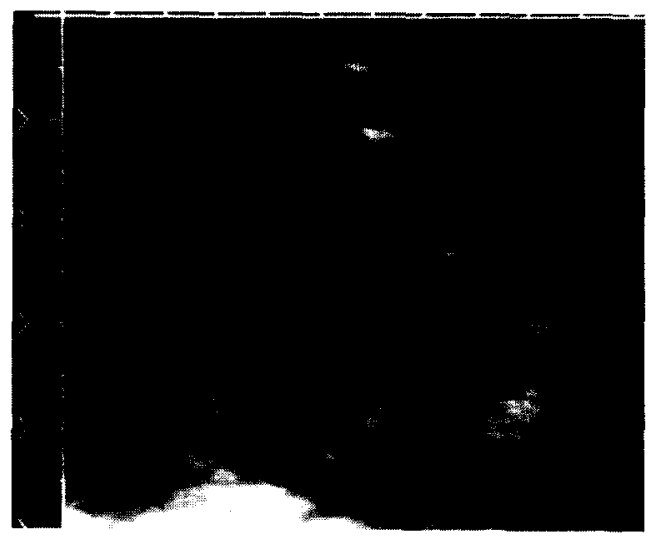

(b)

Fig. 2. (a) Normal human cadaver kidney demonstrating areas of increased echogenicity (arrows) in the cortex where the medullary rays and interlobular arteries would lie perpendicular to the sound field similar to the sheep kidney. The right-hand edge is brighter than the left, but both areas are brighter than the intervening cortex. The bright echo on the near surface of the kidney (hollow arrow) is a specular reflection from the capsule and does not relate to the cortical echogenicity. (b) Ninety-degree rotation again showing hyperechoic areas along the left- and right-hand margins of the cortex. These areas are different parts of the cortex than those imaged in 2(a), but the relationship of the echogenic areas to the sound field are the same as in 2(a). The near field specular reflection is again noted (arrow). 


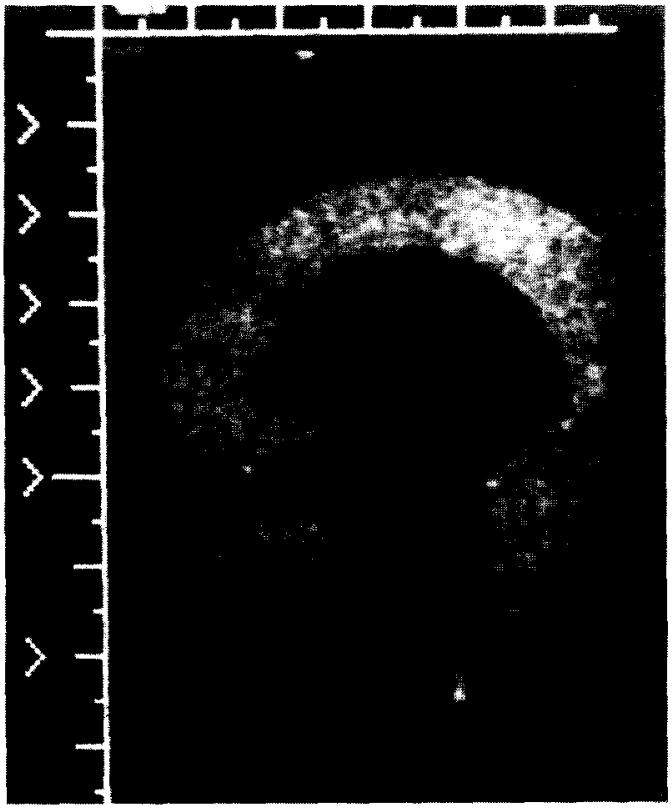

Fig. 3. Slice through a uniform cardiac phantom using the 5 $\mathrm{MHz}$ linear array demonstrating only progressive drop off of the echogenicity in the far field due to attenuation. The phantom is otherwise basically uniform. Note that no local increases are demonstrated at the levels of best focus (sites corresponding to the carets displayed along the left-hand margin of the image).

nonspecific sign of diffuse pathology (Rosenfield et al., 1980; Rosenfield and Siegel, 1981; Hricak et al., 1982). Therefore, it is imperative to understand the normal distribution of echoes in the renal cortex before making inferences about pathological conditions. Cortical anisotropy is another property of renal

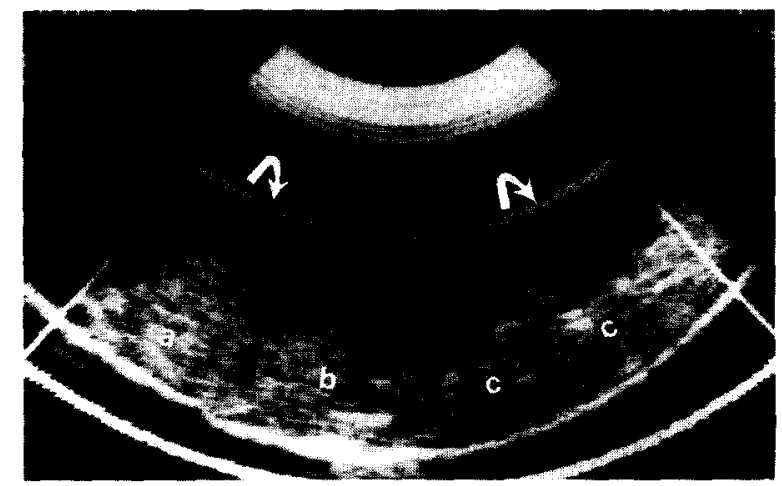

Fig. 4. 7.5 MHz scan of samples of cortex from a sheep's kidney lying side-by-side to determine their relative echogenicities. The two small blocks of cortex on the left labelled $\mathrm{a}$ and $\mathrm{b}$ are positioned so that their cortical structures are oriented perpendicular to the sound field, while the larger block (c) has its cortex aligned parallel to the field. A truncated pie-shaped region (curved arrows) is seen surrounding the samples. This area was digitized for analysis. tissue that is now recognizable and thus might prove useful in the diagnosis of renal pathology by either its presence or absence.

It is not surprising that there would be a significant anisotropy visible in the renal cortex. The medullary rays, which are composed of the collecting tubules, the thick ascending limbs, and terminal straight portions of the proximal convoluted tubules are grossly visible as striations in the renal cortex perpendicular to the capsule (Fig. 5) (Hepinstall, 1974). They are accompanied by interlobular arteries which define the margins of the lobule. The intralobular areas between the medullary rays and the interlobular arteries are taken up mainly by glomeruli and proximal and distal convoluted tubules.

The medulla is composed of two zones: (1) the inner zone or papilla, which contains collecting ducts, their limbs, and loops of Henle and the vasa recta, and (2) the outer zone of the medulla, which has its own outer and inner zones: the outer zone contains the terminal straight portions of the proximal convoluted tubules with the inner zone composed of the thick ascending limbs and collecting tubules (Hepinstall, 1974). Although these tubules are directionally oriented toward the papillae, we could not demonstrate any anisotropy or any relatively strong backscatter in the medulla. This could be because these tubules tend to be thinner with flatter epithelial cells than the tubules in the cortex. They, therefore, could be too small to resolve and may present too small a surface to produce a significant reflection. The tubules also may be too tightly packed, or too well matched in acoustic impedance with their contents and other medullary tissues. Even

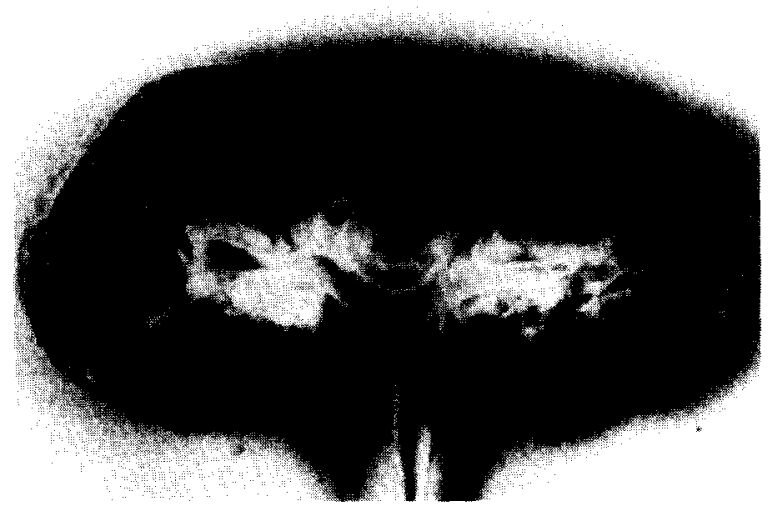

Fig. 5. A longitudinal anatomic section through a human cadaver kidney demonstrates the prominent striations in the renal cortex that correspond to the medullary rays. These striations are clearly perpendicular to the renal capsule and largely produce the anisotropy seen on the ultrasound images. (This image was reproduced with permission from Heptinstall, 1974, p. 2.) 
if there were anisotropy of more than $2-3 \mathrm{~dB}$ in backscatter from medullary tissues, it might not have been detected here because of the shape and small size of the medullary pyramids and the fact that the surrounding tissues are more echogenic than the medulla. It is difficult to know whether partial volume effects are the cause of any differences in the echogenicity of the medullary pyramids in a given image.

The observation that the inclusion angles for the kidneys are greater than for a flat reflector is to be expected, since medullary rays with chains of glomeruli should not constitute specular reflectors. The glomeruli are approximately spherical in shape with diameters on the order of .1-.2 mm. Since the concentration of glomeruli in the cortices of the kidneys of infants is higher than in adults (Haller et al., 1982; Dunnill and Halley, 1973), the aniostropic effect would be diminished. We have, in fact, observed this in vivo.

As mentioned earlier, there have definitely been other instances in which tissue anisotropies could be demonstrated by ultrasound. The heart, tendons, and skeletal musculature are well-described examples (Fei and Shung, 1985; Miller et al., 1983; Mol and Breddels, 1982; Ophir et al., 1982; Cady et al., 1983; Fornage, 1986; Fornage, 1987), and the recent demonstration of the orientationally dependent imaging of nerve tracts in infant heads appears to be another example of an ultrasonically visible tissue anisotropy (Bowerman and DiPietro, 1987). In addition, it is of importance to recognize significant anisotropic properties in tissue, since many of the tissue characterization techniques now employed assume isotropic backscatter and could be very adversely effected by directionally dependent backscatter (Nicholas, 1977).

It is interesting to note that the approximately 5 $\mathrm{dB}$ anisotropy in echogenicity we demonstrated in the renal cortex is almost exactly equal to the anisotropic attenuation difference measured by Ophir et al. (1982) in the quadriceps femoris muscles of 10 normal volunteers. The significance of this association, if any, will require further study.

Acknowledgements-We are indebted to Milton Dupuy, M.S.E.E., for writing and testing of the relative echogenicity software.

Development of the quantitative techniques and apparatus was funded in part by PHS grant number 2 ROI HD 17243 from the National Institute for Human Growth and Development, DHHS.

\section{REFERENCES}

Bowerman R. A. and DiPietro M. A. (1987) Erroneous sonographic identification of lateral ventricles: Relationship to the echogenic periventricular "blush". Am. J. Neurorad. 8, 661-664.
Cady E. B., Gardener J. E. and Edwards R. H. T. (1983) Ultrasonic tissue characterization of skeletal muscle. Eur. J. Clin. Invest. $13,469-473$.

Chivers R. C. (1981) Tissue characterization. Ultrasound in Med. \& Biol. 7, 1-20.

Cook J. H. III, Rosenfield A. T. and Taylor K. J. W. (1977) Ultrasonic demonstration of intrarenal anatomy. Am. J. Roentgenol. 129, 831-835.

Cramer B. C., Jequier S. and De Chadarevian (1986). Factors associated with renal parenchymal echogenicity in the newborn. $J$. Ultrasound Med. 5, 633-638.

Dunnill M. S. and Halley W. (1973) Some observations on the quantitative anatomy of the kidney. J. Pathol. 110, 113-121.

Fei D. Y. and Shung K. K. (1985) Ultrasonic backscatter from mammalian tissues. J. Acoust. Soc. Am. 78, 871-886.

Fornage B. (1986) Achilles tendon. US examination. Radiology $159,759-764$.

Fornage B. (1987) The hypoechoic normal tendon-a pitfall. $J$. Ultrasound Med. 6, 19-22.

Haller J. O., Berdon W. E. and Friedman A. P. (1982) Increased renal cortical echogenicity: A normal finding in neonates and infants. Radiology 142, 173-174.

Heptinstall R. H. (1974) Pathology of the Kidney, Vol. 1, 2nd edn, pp. 1-50. Little, Brown and Company, Boston.

Hricak H., Cruz C., Romanski R. et al. (1982) Renal parenchymal disease: sonographic-histologic correlation. Radiology 144, 141-147.

Hricak H., Slovis T. L., Callen C. W., Callen P. W. and Romanski R. N. (1983) Neonatal kidneys: Sonographic anatomic correlation. Radiology 147, 699-702.

Marchal G., Verbeken E., Oyen R. et al. (1986) Ultrasound of the normal kidney: a sonographic, anatomic, and histologic correlation. Ultrasound in Med. \& Biol. 12, 999-1009.

Meyer C. R., Dupuy M. E., Absalom K. et al. (1985) Preliminary results regarding the correlation of fetal lung maturity with quantitative parameters estimated from digitized RF ultrasound. J. Ultrasound Med. 4(suppl), 98.

Miller J. G., Perez J. E., Mottley J. C. et al. (1983) Myocardial tissue characterization: An approach based on quantitative backscatter and attenuation. Proc. IEEE Ultrason. Symp. CH 1947-1, 782-793.

Mol C. R. and Breddels P. A. (1982) Ultrasound velocity in muscle. J. Acoust. Soc. Am. 71, 455-461.

Nicholas D. (1977) Orientation and frequency dependence of backscattered energy and its clinical application. In Advances in Ultrasound in Biomedicine, Vol. 1. (Edited by D. N. White) pp. 29-54. Research Studies Press, Oregon.

Nicholas D. (1979) Ultrasonic diffraction analysis in the investigation of liver disease. Br. J. Radiol. 52, 949-961.

O'Donnell M., Bauwens D., Mimbs J. W. and Miller J. G. (1979) Broadband integrated backscatter: An approach to spatially localized tissue characterization in vivo. Proc. IEEE Ultrason. Symp. 79 CH 1482-9, 175-178.

Ophir J., Maklad N. F. and Bigelow R. H. (1982) Ultrasonic attenuation measurements of in vivo human muscle. Ultrasonic Imaging 4, 290-295.

Pierce A. D. (1981) Acoustics-An introduction to its physical principles and applications, pp. 428-433. McGraw-Hill, New York.

Rosenfield A. T. and Siegel N. J. (1981) Renal parenchymal disease: histopathologic-sonographic correlation. Am. J. Roentgenol. 137. 793-798.

Rosenfield A. T., Taylor K. J. W., Crade M. and DeGraaf C. S. (1978) Anatomy and pathology of the kidney by gray scale ultrasound. Radiology 128, 737-744.

Rosenfield A. T., Taylor K. J. W. and Jaffe C. C. (1980) Clinical applications of ultrasound tissue characterization. Rad. Clin. N. Am. 18, 31-58. 